\title{
DOSES DE FÓSFORO E CRESCIMENTO RADICULAR DE CULTIVARES DE ARROZ DE TERRAS ALTAS ${ }^{(1)}$
}

\author{
CARLOS ALEXANDRE COSTA CRUSCIOL ${ }^{(2,3)}$; MUNIR MAUAD ${ }^{(2)}$; RITA DE CASSIA \\ FELIX ALVAREZ ${ }^{(2)}$; EDUARDO DO VALLE LIMA ${ }^{(2)}$; CARLOS SÉRGIO TIRITAN ${ }^{(2)}$
}

\begin{abstract}
RESUMO
O estudo de crescimento radicular de arroz de terras altas em função da disponibilidade de fósforo é, praticamente, inexistente. O objetivo deste trabalho foi avaliar o crescimento de diversas cultivares de arroz de terras altas em diferentes condições de disponibilidade de fósforo. $\mathrm{O}$ experimento foi desenvolvido em casa de vegetação, em delineamento inteiramente casualizado, em esquema fatorial 4 x 4, com quatro doses de P $\left(0,50,100\right.$ e $\left.200 \mathrm{mg} \mathrm{dm}^{-3}\right)$ e quatro cultivares: Maravilha (grupo moderno), IAC 201, IAC 202 e Carajás (grupo intermediário). A cultivar Carajás possui sistema radicular que melhor se desenvolveu sob baixa disponibilidade de P. Sob baixa disponibilidade de P as cultivares IAC 201 e IAC 202 priorizaram o desenvolvimento do sistema radicular em relação a parte aérea. A cultivar Maravilha requer níveis elevados de fósforo para atingir o máximo desenvolvimento. Sob baixa disponibilidade de fósforo as cultivares de arroz diminuíram o diâmetro radicular.
\end{abstract}

Palavras-chave: adubação, comprimento radicular, matéria seca, oryza sativa L.

\section{ABSTRACT \\ PHOSPHORUS DOSES AND ROOT GROWTH OF UPLAND RICE}

Very little is known of phosphorus availability for upland rice. The objective of this work was to evaluate root growth of upland rice cultivars in different conditions of phosphorus availability. The experiment was carried out in greenhouse, in a completely randomized design, as a $4 \times 4$ factorial, with four doses of $\mathrm{P}\left(0,50,100\right.$ and $\left.200 \mathrm{mg} \mathrm{dm}^{-3}\right)$ and four cultivars: Maravilha (modern group), IAC 201, IAC 202 and Carajás (middle group). The Carajás root system grew more in low availability of $\mathrm{P}$ than other cultivars. In low availability of P the cultivars IAC 201 and IAC 202 priorized root system growth to aerial growth. The Maravilha cultivar needs high Plevels to reach maximum growth. In low availability of $\mathrm{P}$ the upland rice cultivars decreased root diameter.

Key words: fertilization, root length, dry matter, oryza sativa L.

$\left({ }^{1}\right)$ Recebido para publicação em 9 de agosto de 2004 e aceito em 29 de agosto de 2005.

$\left({ }^{2}\right)$ Departamento de Produção Vegetal (DPV) FCA/UNESP. Caixa Postal 237, 18603-970 Botucatu (SP). E-mail: crusciol@fca.unesp.br; mauad@laser.com.br

$\left({ }^{3}\right)$ Bolsista CNPq. 


\section{INTRODUÇÃO}

Atualmente, no Brasil, cerca de um terço da produção de grãos de arroz origina-se de lavouras cultivadas no ecossistema de terras altas. Essas áreas, por sua vez, correspondem a dois terços da área total cultivada com o cereal, e tem o sistema de sequeiro como principal modalidade. A quase totalidade do arroz produzido no ecossistema de terras altas é cultivado na região do cerrado brasileiro. Os solos dessa região, em sua maioria, têm baixa capacidade de retenção de água, baixo teor de $\mathrm{P}$ e alto teor de alumínio, sendo os três fatores que mais limitam a produção no cerrado.

Dentre os macronutrientes primários, o P é o de menor exigência para a cultura do arroz, porém é o de maior exportação percentual no produto colhido (FORNASIERI e FORNASIERI FILHO, 1993) e o mais deficiente na maioria dos solos brasileiros, devido ao baixo teor natural e a alta capacidade de fixação (FAGERIA, 1999).

Em diversas culturas tem sido demonstrado que o $\mathrm{P}$ exerce grande influência sob o crescimento radicular, no entanto, na cultura do arroz de terras altas os estudos são, praticamente, inexistentes. No arroz cultivado no ecossistema de várzea, a literatura é abundante, porém, as cultivares possuem sistema radicular mais superficial e denso, morfologicamente diferente das cultivares de arroz utilizadas no ecossistema de terras altas (Nemoto e Shigenori, 1994).

O contato íon raiz para o $\mathrm{P}$ dá-se por difusão, movimentado-se pouco e vagarosamente no solo, obrigando a um crescimento constante do sistema radicular (Rosolem, 1995). A difusão de P no solo é mais limitante para a absorção de $\mathrm{P}$ do que a velocidade de absorção radicular (ArAújo, 2000). Segundo Rosolem et al. (1994), os principais fatores que afetam a absorção de $\mathrm{P}$ pela plantas são a taxa de crescimento radicular, a concentração do $\mathrm{P}$ na solução do solo e raio médio das raízes.

Embora o padrão de enraizamento esteja sob controle genético, o crescimento da raízes pode ser modificado por características químicas e físicas do solo (TAYLOR E ARKIN, 1981).

Estudos desenvolvidos por KLEPKER E ANGHinONi (1996) E Rosolem (1995) permitem inferir maior crescimento de raízes de milho, tanto em massa como em comprimento, quanto maior a disponibilidade de $\mathrm{P}$ para as plantas. Vários autores relatam que além dos parâmetros fisiológicos da absorção (Imax, Km, Vmax), a capacidade de absorção de nutrientes está relacionada com o comprimento radicular (BARBER, 1984; TEO et al., 1995; ROSOlEM et al., 1999), especialmente para aqueles nutrientes de baixa mobilidade no solo como o P.
Segundo Vilela e Anghinoni (1984), baixa concentração de $\mathrm{P}$ no solo provoca diminuição no comprimento e o engrossamento das raízes de soja, entretanto, em milho, SHenk E BArber (1977) verificaram redução no raio médio da raiz com a diminuição do teor de fósforo no solo.

BARBER (1984) observou aumento no crescimento radicular de cultivares de milho com o suprimento de P no solo, entretanto, esse efeito pareceu ser dependente das cultivares (SHENK E BARBER, 1980). Para elementos com baixa taxa de difusão no solo, como os fosfatos, plantas com maior superfície radicular possuem maior capacidade para absorção dos nutrientes do solo (TeO et al., 1995), em virtude da proximidade entre a superfície absortiva da raiz e o nutriente ser muito importante (HARPER et al., 1991; Rosolem, 1995).

O objetivo deste trabalho foi avaliar a influência da adubação fosfatada no crescimento radicular de cultivares de arroz de terras altas.

\section{MATERIAL E MÉTODOS}

O experimento foi desenvolvido em casa de vegetação no Departamento de Produção Vegetal da Faculdade de Ciências Agronômicas - UNESP, Campus de Botucatu, São Paulo. Utilizaram-se amostras de Latossolo Vermelho Distroférrico com as seguintes características : $\mathrm{pH}\left(\mathrm{CaCl}_{2}\right)=4,1 ; \mathrm{M} . \mathrm{O}=18,0$

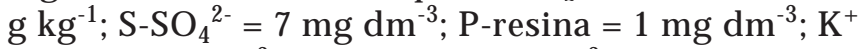
$=0,1 \mathrm{mmol}_{\mathrm{c}} \mathrm{dm}^{-3} ; \mathrm{Ca}^{++}=4 \mathrm{mmol}_{\mathrm{c}} \mathrm{dm}^{-3} ; \mathrm{Mg}^{++}=1 \mathrm{mmol}_{\mathrm{c}}$ $\mathrm{dm}^{-3} ; \mathrm{H}+\mathrm{Al}=75 \mathrm{mmol}_{\mathrm{c}} \mathrm{dm}^{-3} ; \mathrm{CTC}=58 \mathrm{mmol}_{\mathrm{c}} \mathrm{dm}^{-3} \mathrm{e}$ $\mathrm{V}=6,4 \%$, determinados conforme RaIj et al. (2001).

Antes da aplicação dos tratamentos o solo utilizado recebeu calcário dolomítico $(\mathrm{PRNT}=93 \%)$ para elevar a saturação por bases a $70 \%$. Os tratamentos foram constituídos de quatro doses de $\mathrm{P}$ $\left(0,50,100\right.$ e $\left.200 \mathrm{mg} \mathrm{dm}^{-3}\right)$ utilizando-se como fonte superfosfato simples e quatro cultivares: Maravilha (grupo moderno), IAC 201, IAC 202 e Carajás (grupo intermediário). Cada unidade experimental constituiu -se de um vaso plástico de $10 \mathrm{~L}$, contendo $8 \mathrm{~kg}$ de amostra de solo. Cada vaso recebeu $100 \mathrm{mg} \mathrm{dm}^{-3} \mathrm{de}$ $\mathrm{N}\left(50 \mathrm{mg} \mathrm{dm}^{-3}\right.$ na semeadura e $50 \mathrm{mg} \mathrm{dm}^{-3} \mathrm{em}$ cobertura no início do estádio de perfilhamento) e 150 $\mathrm{mg} \mathrm{dm}^{-3}$ de $\mathrm{K}$, utilizando-se uréia e cloreto de potássio como fonte de $\mathrm{N}$ e K respectivamente, juntamente com a aplicação dos tratamentos com P.

Foram semeadas cinco sementes por vasos e uma semana após a emergência foi realizado o desbaste, deixando-se duas plantas por vasos. Aos 79 dias após a emergência, quando as plantas se 
encontravam no estádio de diferenciação floral, foi feita colheita das plantas. As plantas foram seccionadas no colo separando a parte aérea do sistema radicular.

A parte aérea das plantas foi lavada em água corrente e a seguir submetida à secagem, em estufa com circulação forçada de ar, a $65^{\circ} \mathrm{C}$, e pesada para a determinação da matéria seca da parte aérea.

As raízes foram separadas do solo por lavagem em água corrente sobre peneira com malha de $0,5 \mathrm{~mm}$. Após a lavagem de todo o sistema radicular de cada unidade experimental, foi tomada uma amostra constituída de $30 \%$ do peso fresco, extraída no sentido do comprimento, ou seja, do ponto de surgimento das raízes adventícias, no colo da planta, até a extremidade do sistema radicular; em seguida, as amostras foram armazenadas em coletor universal, com capacidade de $100 \mathrm{~mL}$, em solução alcoólica 70 $\%$, e acondicionada em ambiente refrigerado, para posterior análise.

As variáveis comprimento, superfície e diâmetro médio das raízes foram avaliadas em Scanner, desenvolvido para esse fim, acoplado a um computador contendo o Software WinRhizo, que utiliza como princípio o método proposto por TENNANT (1975). Os $70 \%$ restantes do sistema radicular juntamente com os $30 \%$ utilizados nas determinações morfológicas foram secos em estufa a $65{ }^{\circ} \mathrm{C}$, onde, posteriormente

$1 \mathrm{~A}$

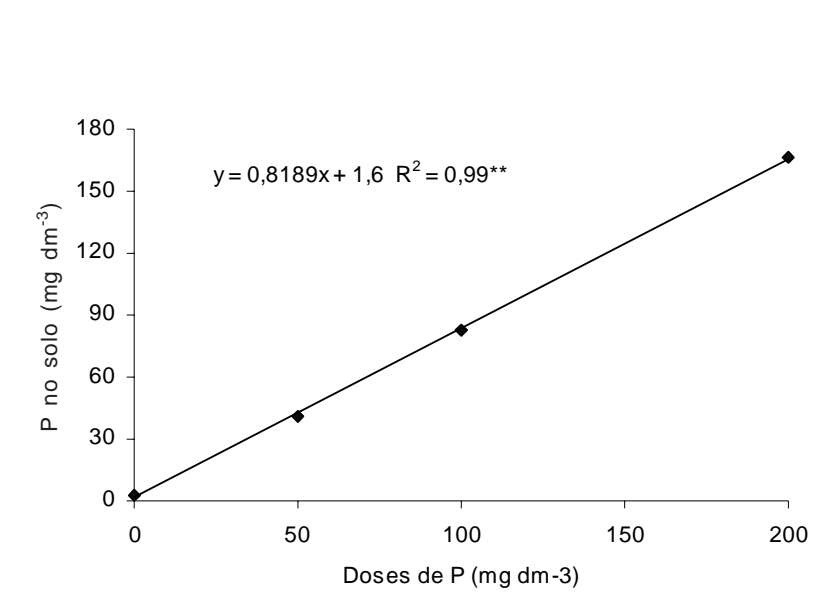

foi determinada a massa seca radicular (mg planta ${ }^{1}$ ). Determinou-se a matéria seca total por meio do somatório da matéria seca da parte aérea e do sistema radicular.

O delineamento experimental foi inteiramente casualizado em esquema fatorial $4 \times 4$, com quatro repetições. Os dados foram submetidos à analise de variância e as médias entre cultivares comparadas pelo Teste DMS a 5\% de significância e o efeito das doses de fósforo e os desdobramentos das interações doses $\mathrm{X}$ cultivares pela análise de regressão.

\section{RESULTADOS E DISCUSSÃO}

O teor de P no solo foi incrementado de forma linear e significativa (Figura1A) com o aumento das doses do elemento, corroborando com os resultados encontrados por FAGERIA et al. (1999). Nota-se que na dose mais elevada ( $\left.200 \mathrm{mg} \mathrm{dm}^{-3}\right)$, o teor de $\mathrm{P}$ no solo não atingiu o teor estimado; provavelmente, esteja relacionado ao processo de precipitação que, segundo LiN et al. (1983), ocorre quando há alta concentração de P no meio.

Na matéria seca da parte aérea, o teor de P não diferiu estatisticamente entre as cultivares e não houve efeito da interação entre cultivares e doses de P (Figura 1 B). A aplicação de $\mathrm{P}$ incrementou o teor do elemento na parte aérea de forma quadrática, atingindo o maior valor na dose calculada de $193 \mathrm{mg} \mathrm{dm}^{-3}$ de P (Figura 1B).

1B
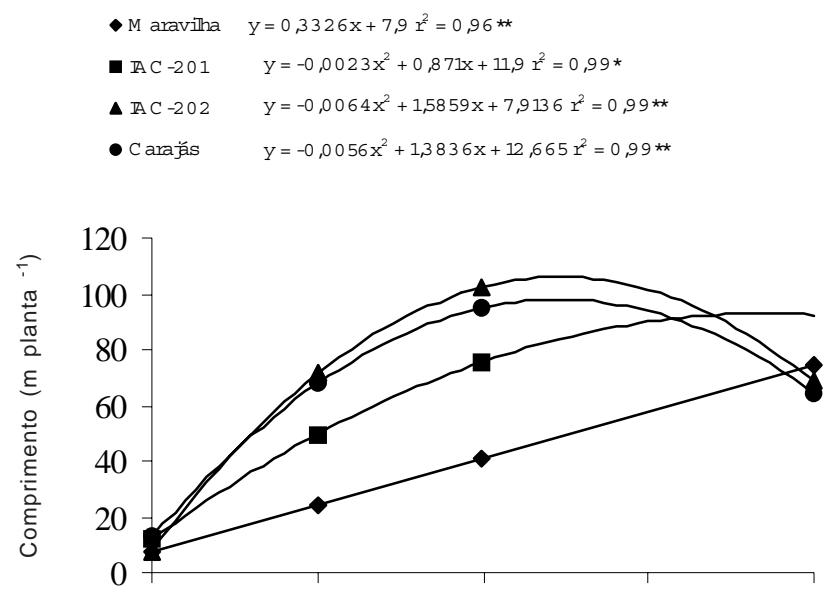

Figura 1. Teor de fósforo no solo (A) e na planta (B) de arroz de terras altas em função da adubação fosfatada (** significativo a $1 \%$ de probabilidade pelo teste $\mathrm{F}$ ). 
As produções de matéria seca radicular, da parte aérea e total, foram influenciadas pelas doses de $\mathrm{P}$ e houve interação entre doses e cultivares. Analisando o desdobramento da interação (Figuras $2 \mathrm{~A}, 2 \mathrm{~B}$ e $2 \mathrm{C}$ ) doses de fósforo dentro de cultivares, verificou-se que em todas as cultivares houve efeito positivo à adubação fosfatada. Nas cultivares Carajás, IAC 202 e IAC 201 houve aumento da matéria seca radicular de forma quadrática, com as máximas produções nas doses calculadas de 124, 155 e $128 \mathrm{mg}$ $\mathrm{dm}^{-3}$ de $\mathrm{P}$ respectivamente (Figura $2 \mathrm{~A}$ ). Na 'Maravilha' a adubação fosfatada influenciou linearmente, permitindo constatar que é cultivar mais responsiva em relação às demais (Figura 2A).

$\mathrm{Na}$ ausência de adubação fosfatada constatou-se que as cultivares IAC 201 e IAC 202 (Tabela 1) priorizaram o desenvolvimento radicular em relação a parte aérea, uma vez que nessa condição houve maior crescimento das raízes. No entanto, constatou-se que a cultivar Carajás possui maior adaptação à condição de baixa disponibilidade de $\mathrm{P}$ em vista da maior produção de matéria seca, tanto radicular quanto da parte aérea. A maior produção de matéria seca radicular em relação à da parte aérea, observada nas cultivares IAC, em condições de baixa disponibilidade de fósforo, é uma estratégia da planta para aumentar a capacidade de aquisição do elemento. Os fotoassimilados produzidos na parte aérea são translocados para o sistema radicular, a fim de aumentar a área de exploração pelas raízes como observado, também, em algodão por Rosolem et al. (1999) e feijão por Araújo ( 2000).

Quando ocorreu o fornecimento de fósforo, o acúmulo de matéria seca da parte aérea seguiu o mesmo comportamento constatado para matéria seca radicular (Figuras 2A e 2B), refletindo na matéria seca total (Figura 2C). Para a cultivar Maravilha a aplicação de fósforo incrementou linearmente essas três variáveis, evidenciando maior exigência e responsividade à melhoria da fertilidade do solo pelas cultivares do grupo moderno em relação às do grupo intermediário e tradicional (FAGERIA et al., 1995), ou seja, em muitas situações, necessitam de níveis mais elevados para atingirem a máxima produtividade. Nas cultivares Carajás, IAC 202 e IAC 201, verificou-se máxima produção com as doses médias calculadas de, 124, 155 e $128 \mathrm{mg} \mathrm{dm}^{-3}$ para matéria seca radicular, 139, $141 \mathrm{e}$ $182 \mathrm{mg} \mathrm{dm}^{-3}$ para matéria seca da parte aérea e 134, 136 e $171 \mathrm{mg} \mathrm{dm}^{-3}$ para matéria seca total, respectivamente. $\mathrm{O}$ efeito diferenciado quanto ao acúmulo de matéria seca da parte aérea entre cultivares de arroz de terras altas, decorrente da adubação fosfatada, também foi constatado por outros pesquisadores (FAGERIA e ZiMERMANN, 1979; FAGERIA et al., 1982; FAGERIA et al., 1988; FAGERIA, 1991; FAGERIA et al., 1995).
O diâmetro radicular foi influenciado apenas pelas doses de $P$, enquanto $O$ comprimento, superfície e volume radicular foram influenciados pelos fatores doses de P e cultivares, e pela interação. Esses resultados corroboram com os de Taylor e Arkin (1981) e Glass (1990), que relataram a alteração no cres-cimento das raízes em função da fertilidade do solo.

Assim como para produção de matéria seca, o efeito de doses de P nas cultivares estudadas, referente ao comprimento, superfície e volume radicular, foi muito semelhante, ou seja, incremento com comportamento quadrático das cultivares Carajás, IAC 202 e IAC 201, e linear da Maravilha (Figura 3). Nas cultivares Carajás e IAC 202 ocorreram os maiores valores, em relação aos demais cultivares, para comprimento, superfície e volume, nas doses calculadas de 112, 125 e $128 \mathrm{mg} \mathrm{dm}^{-3}$ e 132, 123 e 128 $\mathrm{mg} \mathrm{dm}{ }^{-3}$, respectivamente. Na cultivar IAC $201 \mathrm{o}$ incremento foi significativo até as doses calculadas de 218,165 e $143 \mathrm{mg} \mathrm{dm}^{-3}$ respectivamente, para comprimento, superfície e volume, enquanto na 'Maravilha' o incremento foi linear.

No entanto, pode-se verificar, pelas equações (Figuras 2 A, B e C), que a cultivar Carajás seguida da IAC 201 possuem maior habilidade, em relação às demais, em se desenvolverem na ausência de adubação fosfatada, sendo melhor constatado na Tabela 1. Esse comportamento está associado à maior adaptação à condições adversas que as cultivares do grupo intermediário possuem em relação ao grupo moderno; assim como a capacidade do sistema radicular em alterar sua configuração geométrica, de forma a melhor explorar o solo em busca de nutrientes ou de adquirir os recursos necessários de maneira mais eficiente (Tiffney e NiKlas, 1985).

As maiores produções de matéria seca radicular, da parte aérea e total das cultivares Carajás e IAC 202, que foram bem superiores às das demais cultivares, em torno da dose de 130 $\mathrm{mg} \mathrm{dm^{-3 }}$, provavelmente, foram devidas ao maior desenvolvimento radicular obtido próximo a essa dose. As cultivares Carajás e IAC 201 se adaptam melhor às condições de baixa disponibilidade de $P$ que a IAC 202 e a Maravilha (Tabela 1). Contudo, o desenvolvimento das cultivares Carajás e IAC 202, em relação as demais, é mais intenso com a aplicação das menores doses de $\mathrm{P}$ e, também, atingem o máximo crescimento radicular e acúmulo de matéria seca com doses menores (Figuras 3A, 3B, 3C, 2A, 2B e 2C). 

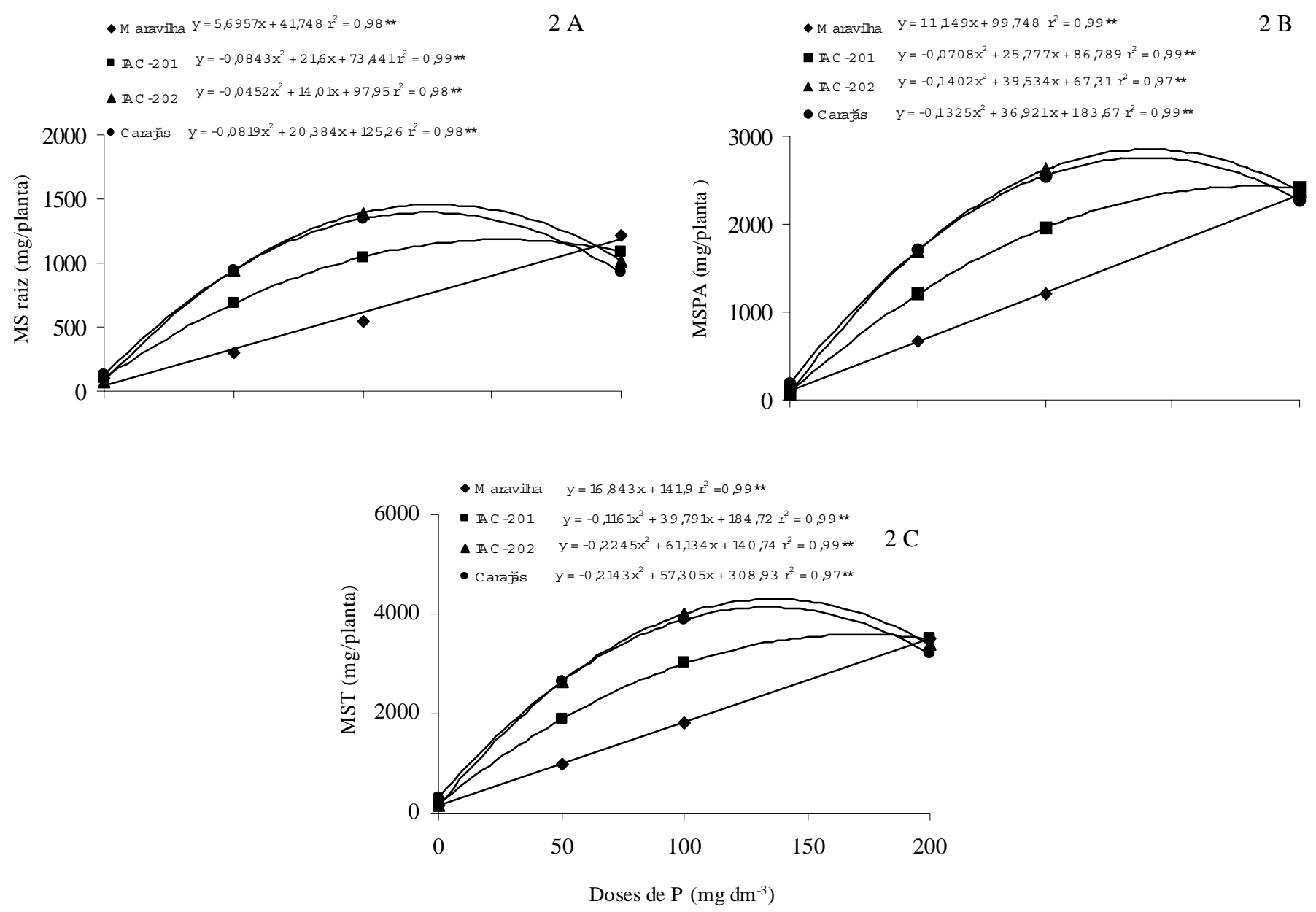

Figura 2. Matéria seca de raiz (MS raiz) (A), da parte aérea (MSPA) (B) e total (MST) (C) de cultivares de arroz de terras altas, colhidas no estádio de diferenciação floral em função da doses de adubo fosfatado (** significativo a $1 \%$ de probabilidade pelo teste F).

Tabela 1. Comprimento, superfície, volume e matéria seca radicular e matéria seca da parte aérea de cultivares de arroz de terras altas na dose de $0 \mathrm{mg} \mathrm{dm}^{-3}$ de fósforo (teor de $\mathrm{P}$ no solo na dose de $0 \mathrm{mg} \mathrm{dm}^{-3}$ de fósforo após aplicação dos tratamentos $=3$ $\mathrm{mg} \mathrm{dm^{-3 } )}$

\begin{tabular}{|c|c|c|c|c|c|}
\hline Cultivares & Comprimento & Superfície & Volume & $\begin{array}{c}\text { Matéria seca } \\
\text { radicular }\end{array}$ & $\begin{array}{c}\text { Matéria seca } \\
\text { parte aérea }\end{array}$ \\
\hline & $\mathrm{m} /$ planta & $\mathrm{cm}^{2} /$ planta & $\mathrm{cm}^{3} /$ planta & g/planta & g/planta \\
\hline Maravilha & $0,79 \mathrm{~b}$ & $105 \mathrm{c}$ & $0,66 \mathrm{c}$ & $97 \mathrm{~b}$ & $100 \mathrm{~b}$ \\
\hline IAC 201 & $1,19 \mathrm{ab}$ & $138 \mathrm{~b}$ & $1,19 \mathrm{~b}$ & $98 \mathrm{~b}$ & $87 \mathrm{~b}$ \\
\hline IAC 202 & $0,79 \mathrm{~b}$ & $0,89 \mathrm{c}$ & $0,79 \mathrm{c}$ & $73 \mathrm{c}$ & $67 c$ \\
\hline Carajás & $1,27 \mathrm{a}$ & $169 \mathrm{a}$ & $1,68 \mathrm{a}$ & $125 \mathrm{a}$ & $184 \mathrm{a}$ \\
\hline
\end{tabular}

Médias seguidas de letras diferentes na vertical diferem estatisticamente pelo teste DMS a 5\% de probabilidade. 
O diâmetro radicular (Figura 3 D) aumentou com as doses de fósforo, ajustando-se a uma função quadrática, tendo atingido o máximo valor na dose calculada de $143 \mathrm{mg} \mathrm{dm}^{-3}$. Comportamento semelhante foi constatado por SHENK E BARBER (1977) e seria uma adequação da planta de forma que apresente uma geometria radicular mais favorável à absorção de $\mathrm{P}$, sob baixa disponibilidade. Os resultados de Vilela e Anghinoni (1984) e Hajabbasi e SCHUMARCher (1994) ajudam a explicar a redução do diâmetro sob elevada concentração de $\mathrm{P}$, pois, relatam que sob essa condição há indução à formação de um sistema radicular mais longo e com raízes mais finas, que seriam mais eficientes na absorção de nutrientes do solo.
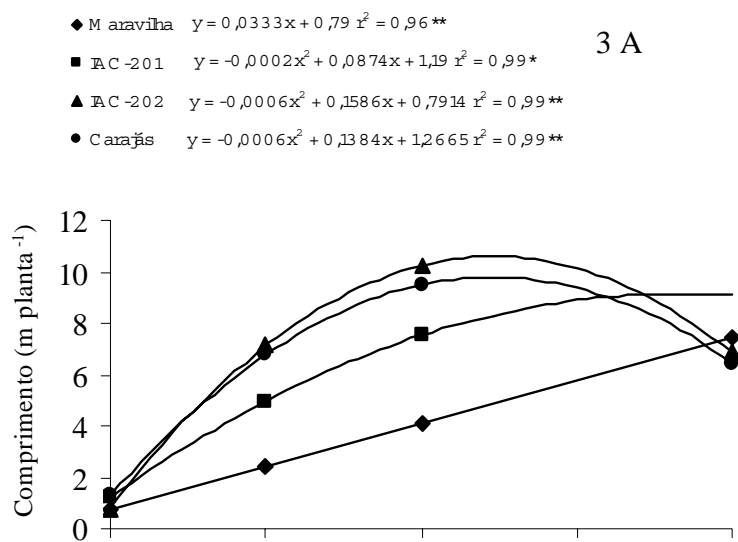

- M aravinha $y=5,4337 x+105,3 x^{2}=0,97$ *

- $\mathbb{A C}-201 \quad \mathrm{y}=-0,0505 \mathrm{x}^{2}+16,744 \mathrm{x}+137,89 \mathrm{r}^{2}=0,99$ **

\ $\mathbb{A C}-202 \quad \mathrm{y}=-0,1051 \mathrm{x}^{2}+26,032 \mathrm{x}+89,079 \mathrm{r}^{2}=0,98$ **

- Carắs $y=-0,0905 x^{2}+22,713 x+168,69 x^{2}=0,99$ **

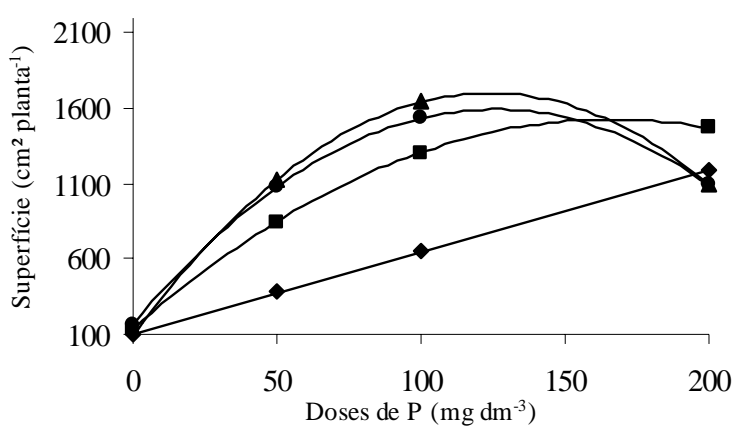

$3 \mathrm{~A}$

$3 \mathrm{~B}$

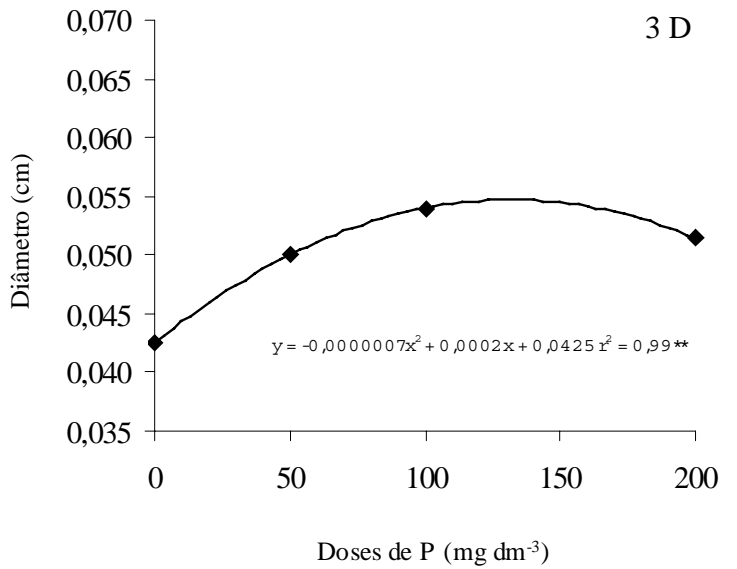

$3 \mathrm{C}$

- $\mathbb{A C}-201 \quad y=-0,0007 x^{2}+0,2443 x+1,1905 x^{2}=0,99$ **

\ $\mathbb{A C}-202 y=-0,0013 x^{2}+0,3348 x+0,7895 x^{2}=0,96$ **

- C arä̆s $y=-0,0012 x^{2}+0,3062 x+1,6795 x^{2}=0,99$ **

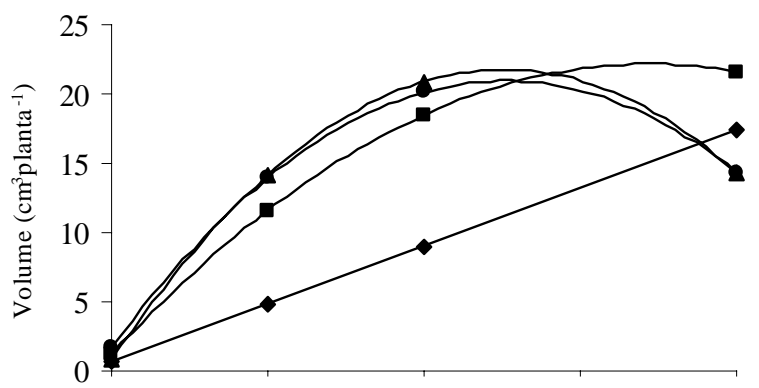

0

Figura 3. Comprimento (A), superfície (B), volume (C) e diâmetro radicular (D) de cultivares de arroz de terras altas colhidas no estádio de diferenciação floral em função da doses de adubo fosfatado (* e ** significativo a $5 \%$ e $1 \%$ de probabilidade pelo teste $\mathrm{F}$ respectivamente).

\section{CONCLUSÕES}

1. A cultivar Carajás possui sistema radicular que melhor se desenvolve sob baixa disponibilidade de $P$.

2. Sob baixa disponibilidade de $\mathrm{P}$ as cultivares IAC 201 e IAC 202 priorizaram o desenvolvimento do sistema radicular em relação à parte aérea.

3. A cultivar Maravilha requer níveis elevados de fósforo para atingir o máximo desenvolvimento.
4. Sob baixa disponibilidade de fósforo as cultivares de arroz diminuíram o diâmetro radicular.

\section{REFERÊNCIAS}

ARAÚJO, A.P. Eficiência vegetal de absorção e utilização de fósforo, com especial referência ao feijoeiro. In: NOVAIS, R.F., ALVAREZ V., V.H., SCHAEFER, C.E.G.R. (Ed.) Tópicos em ciência do solo. Viçosa: SBCS, v.1, 2000. p.163-212. 
BARBER, S.A. Soil nutrient bioavailability: a mechanistic approach. New York: Willey Interscience, 1984. p.

FAGERIA, N.K. Adubação e calagem. In:VIERIA, N.R.A.; SANTOS, A.B.; SANT'ANA, E.P. (Ed). A cultura do arroz no Brasil, Santo Antonio de Goiás: EMBRAPA-CNPAF, 1999. p.329-353.

FAGERIA, N.K. Resposta de cultivares de arroz a fertilizante fosfatado em Latossolo Vermelho-Escuro do Brasil Central. Revista Brasileira de Ciência do Solo, Viçosa, v.15. n. 1, p.63-7, 1991.

FAGERIA, N.K.; BARBOSA FILHO, M.P. Avaliação de cultivares de arroz para maior eficiência na absorção de fósforo. Pesquisa Agropecuária Brasileira, Brasília, v.16, n.6, p.777-82, 1981.

FAGERIA, N.K.; BARBOSA FILHO, M.P.; CARVALHO, J.R.P. Response of upland rice to phosphorus fertilization on an oxisol of Central Brasil Agronomiy Journal, Madison, v.74, p.51-6, 1982.

FAGERIA, N.K.; MORAIS, O.P.; BALIGAR, V.C.; WRIGHT, R.J. Response of rice cultivars to phosphorus supply on an oxisol. Fertilizer Research, Dordrecht, v.16, p.195-206, 1988.

FAGERIA, N.K.; SANT'ANA, E.P.; MORAES, O.P. Resposta de genótipos de arroz de sequeiro favorecido à fertilidade do solo. Pesquisa Agropecuária Brasileira, Brasília v.30 p. 11551161, 1995.

FAGERIA, N.K.; ZIMMERMANN, F.J.P. Interação entre fósforo, zinco e calcário em arroz de sequeiro. Revista Brasileira de Ciência do Solo, Campinas, v.3, n. 2, p. 88-92, 1979.

FORNASIERI FILHO, D.; FORNASIERI, J.L. Manual da cultura do arroz. Jaboticabal: FUNEP, 1993. 221p.

FURLANI, A.M.C.; BATAGLIA, O.C.; FURLANI, P.R.; AZZINI, L.E; CAMARGO, O.B.A. Avaliação de genótipos de arroz quanto à eficiência na utilização de fósforo em solução nutritiva e em solo. Revista Brasileira de Ciência do Solo, Campinas v.7, n.3, p. 291-303, 1983.

GLASS, A.D.M. Íon absorption and utilization: the celular level. In: BALIGAR, V.C., DUNCAN, R.R. (Ed). Crops as enhancers of nutrient use. San Diego: Academic Press, 1990. p.37-64.

HAJABBASI, M.A.; SCHUMAKER, T.E. Phosphorus effects on root growth and development in two maize genotypes. Plant and Soil, Dordrecht, v.158, p.39-46, 1994.

HARPER, J.L.; JONES, M.; SACKVILLE-HAMILTON, N.R. The evolution of roots and the problems of analysing their behaviour. In: ATKINSON, D. (Ed.). Plant root growth: an ecological perspective. Oxford: Blackwell, 1991. p.3-22.

KLEPKER, D.; ANGHINONI, I. Crescimento radicular e aéreo do milho em vasos em função do nível de fósforo e localização do adubo fosfatado. Revista Brasileira de Ciência do Solo, Viçosa v.19, n.3, p. 403-408, 1996.
LIN, C., BUSSCHER, W.J., DOUGLAS, L.A. Multifactor kinetics of phosphate reactions with minerals in acidic soils. I. Modeling and simulation. Soil Science of America Journal, Madsion v.47, p.1097-1103, 1983.

LYNCH, J.; BEEM, J.J.van. Growth and architecture of seeding roots of commom bean genotypes. Crop Science, Madsion v.33, p.1253-1257, 1993.

NEMATO, K.; SHIGENORI, M.; Morphology and anatomy of rice roots with special refence to coordination in organo-and histogenesis. In: BALUSKA, F.; CIAMPOROVÂ, M.; GASPARIKOVÁ, O.; BARLOW, P.W. (Ed.) Structure and function of roots, Dordecht, 1994. p. 75-86.

RAIJ, B. van; ANDRADE, J.C.; CANTARELLA, H.; QUAGGIO, J.A. (Ed.). Análise química para avaliação da fertilidade de solos tropicais. Campinas: Instituto Agronômico, 2001. 285 p.

ROSOLEM, C.A.; ASSIS, J.S.; SANTIAGO, A.D. Root growth and mineral nutrition of corn hybrids as affected by phosphorus and lime. Communications in Soil Science and Plant Analysis, New York, v.25 p. 2491-2499, 1994.

ROSOLEM, C.A. Relações solo-planta na cultura do milho. Jaboticabal: Funep, 1995, 53p.

ROSOLEM, C.A.; WITACKER, J.P.T.; VANZOLINI, S.; RAMOS, V.J. Significance of root growth on cotton nutrition in an acidic low-P soil. Plant and Soil, Dordrecht, v. 212, p.185-190, 1999.

SHENK, N.K.; BARBER, S.A. Root characteristics of corn genotips as related to P uptake. Agronomy Journal, Madison, v.71, p.921-924, 1977.

SHENK, N.K.; BARBER, S.A. Potassium and phosphorus uptake by corn genotips growth in the field as influenced by root characteristics. Plant and Soil, Dordrecht v.54, p.65-76, 1980.

TAYLOR, D.; ARKIN, G.F. Root zone modification fundamentals and alternatives. In: Modifying the root environment to reduce crop stress. St. Joseph: ASAE, 1981. p.3-16.

TEO, Y.H.; BEYROUTY, C.A.; NORMAN, R.J.; GBUR, E.E. Nutrition uptake relationship to root characteristics of rice. Plant and Soil, Dordrecht, v. 171, p.297-302, 1995.

TENNANT, D. A test of a modified line intersect method of estimating root length. Journal of Ecology, Oxford,v.63 p. 995-1001, 1975.

TIFFNEY, B.H.; NIKLAS, K.J. Clonal growth in land plants: a palaeobotanical prespective. In: JACKSON, J.B.C.; BUSS, L.W.; COOK, R.E (Ed). Population biology and evolution of clonal organisms. New Haven: Yale Universiry Press, 1985. p.35-66.

VILELA, L.; ANGHINONI, I. Morfologia do sistema radicular e cinética de absorção de fósforo em cultivares de soja afetadas pela interação alumínio fósforo. Revista Brasileira de Ciência do Solo, Campinas, v.8, n. 1, p.91-96, 1984. 
